The Physics of Evolved Stars

Editors : will be set by the publisher

EAS Publications Series, Vol.?,2021

\title{
INDICATION OF THE HIGH MASS-TRANSFER RATIO IN S-TYPE SYMBIOTIC BINARIES
}

\author{
N. Shagatova ${ }^{1}$ and A. Skopal ${ }^{1}$
}

\begin{abstract}
By modelling $\mathrm{H}^{0}$ column densities in eclipsing S-type symbiotic stars EG And and SY Mus, we derived the wind velocity profile and the corresponding mass-loss rate from their giants. Our analysis revealed a strong enhancement of the wind at the orbital plane.
\end{abstract}

\section{Introduction}

The wind mass transfer in symbiotic binaries is connected with the long-standing problem of a large luminosity of their hot components and an inefficient wind mass transfer from their red giants in the canonical Bondi-Hoyle accretion mechanism. Investigation of the giant wind properties can aid us in a better understanding of the wind mass-transfer mode in these widest interacting binaries.

\section{Method and results}

We investigated a distribution of the neutral hydrogen $\left(\mathrm{H}^{0}\right)$ from the wind of giants in eclipsing S-type symbiotic stars EG And and SY Mus. For this purpose, we used far-UV spectra measured by the IUE and HST satellites, available from their archives. By modelling the Rayleigh attenuation of the continuum around the Ly- $\alpha$ line (Fig. 1, left), we obtained $\mathrm{H}^{0}$ column densities, $n_{\mathrm{H}^{0}}^{\text {obs }}$, at different orbital phases (Fig. 1, right). Some values were supplemented from the literature. Further, we modelled $n_{\mathrm{H}^{0}}^{\mathrm{obs}}$ values taking into account ionization of the giant's wind (Seaquist et al. 1984) and used the inversion method for the column density function according to Knill et al. (1993) to derive the wind velocity profile (WVP). In this way we derived a relation for the total $\mathrm{H}^{0}$ column density, $\tilde{n}_{\mathrm{H}}(b)$, as a function of the orbital phase (or the impact parameter $b$ ), and the WVP, $v(r)$, in the form,

$$
\tilde{n}_{\mathrm{H}}(b)=\frac{n_{1}}{b}+\frac{n_{K}}{b^{K}}, \quad v(r)=\frac{v_{\infty}}{1+\xi r^{1-K}},
$$

Supported by a grant of the Slovak Academy of Sciences, VEGA No. 2/0002/13.

${ }^{1}$ Astronomical Institute of the Slovak Academy of Sciences, Tatranská Lomnica, Slovakia

(c) EDP Sciences 2021

DOI: (will be inserted later) 

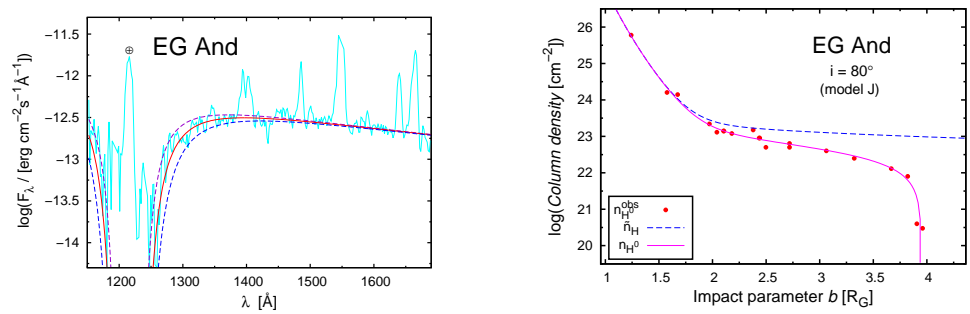

Fig. 1. Left: Far-UV spectrum of EG And attenuated with $n_{\mathrm{H}^{0}}^{\mathrm{obs}}=(1.5+0.7 /-0.5) \times$ $10^{23} \mathrm{~cm}^{-2}$. Right: $n_{\mathrm{H}^{0}}^{\text {obs }}$ values (circles) and our models (solid and dashed line).

where $n_{1}, n_{\mathrm{K}}$ and $K$ are fitting parameters, $v_{\infty}$ is the terminal velocity of the wind, $\xi=\left(n_{K} \lambda_{1}\right) /\left(n_{1} \lambda_{K}\right), \lambda_{1}$ and $\lambda_{K}$ are the eigenvalues of the Abel operator (see Knill et al. 1993). Example of a model and its parameters for EG And are in Fig. 1 and Table 1. Corresponding values of the spherical equivalent of the mass-loss rates, $\dot{M}_{\mathrm{sp}} \approx 10^{-6} M_{\odot} \mathrm{yr}^{-1}$, are a factor of $\approx 10$ larger than total rates measured by independent methods $\left(\approx 10^{-7} M_{\odot} \mathrm{yr}^{-1}\right.$, e.g. Seaquist et al. 1993). This findings suggests that the wind from giants in S-type symbiotic stars is focused towards the binary orbital plane, because both systems are eclipsing, and thus our $n_{\mathrm{H}^{0}}^{\text {obs }}$ values are given by densities at the near-orbital-plane region. According to the model of Nagae et al. (2004), our WVPs correspond to the mass-accretion ratio $15-18 \%$.

Table 1. Resulting parameters, $n_{1}, n_{\mathrm{K}}, K$ and $X^{\mathrm{H}+} ; X^{\mathrm{H}+}$ is the ionization parameter.

\begin{tabular}{lcccccc}
\hline \hline object & $i$ & $n_{1}\left[10^{23}\right]$ & $n_{\mathrm{K}}$ & $K$ & $X^{\mathrm{H}+}$ & $\dot{M}_{\mathrm{sp}}\left[M_{\odot} \mathrm{yr}^{-1}\right]$ \\
\hline EG And & $80^{\circ}$ & 3.87 & $1.15 \times 10^{27}$ & 14 & 1.85 & $1.8 \times 10^{-6}$ \\
\hline
\end{tabular}

\section{Conclusions}

By modelling the $\mathrm{H}^{0}$ column densities around giants in eclipsing S-type symbiotic stars EG And and SY Mus, we determined the WVP and the corresponding $\dot{M}_{\mathrm{sp}}$ from their giants. Our analysis revealed that $\dot{M}_{\mathrm{sp}}$ are a factor of $\approx 10$ higher than observed total $\dot{M}$. This suggests that the giant's wind is significantly enhanced at the orbital plane, where can be effectively accreated onto the hot component. In this way, we indicated a high mass-transfer ratio in S-type symbiotic binaries.

\section{References}

Knill, O., Dgani, R. \& Vogel, M. 1993, A\&A, 274, 1002

Nagae, T., Oka, K., Matsuda, T., Fujiwara, H., Hachisu, I. \& Boffin, H. M. J. 2004, A\&A, 419, 335

Seaquist, E. R., Taylor, A. R. \& Button, S. 1984, ApJ, 284, 202

Seaquist, E. R., Krogulec, M., \& Taylor, A. R. 1993, ApJ, 410, 260 